\title{
UNCLASSFFIED
}
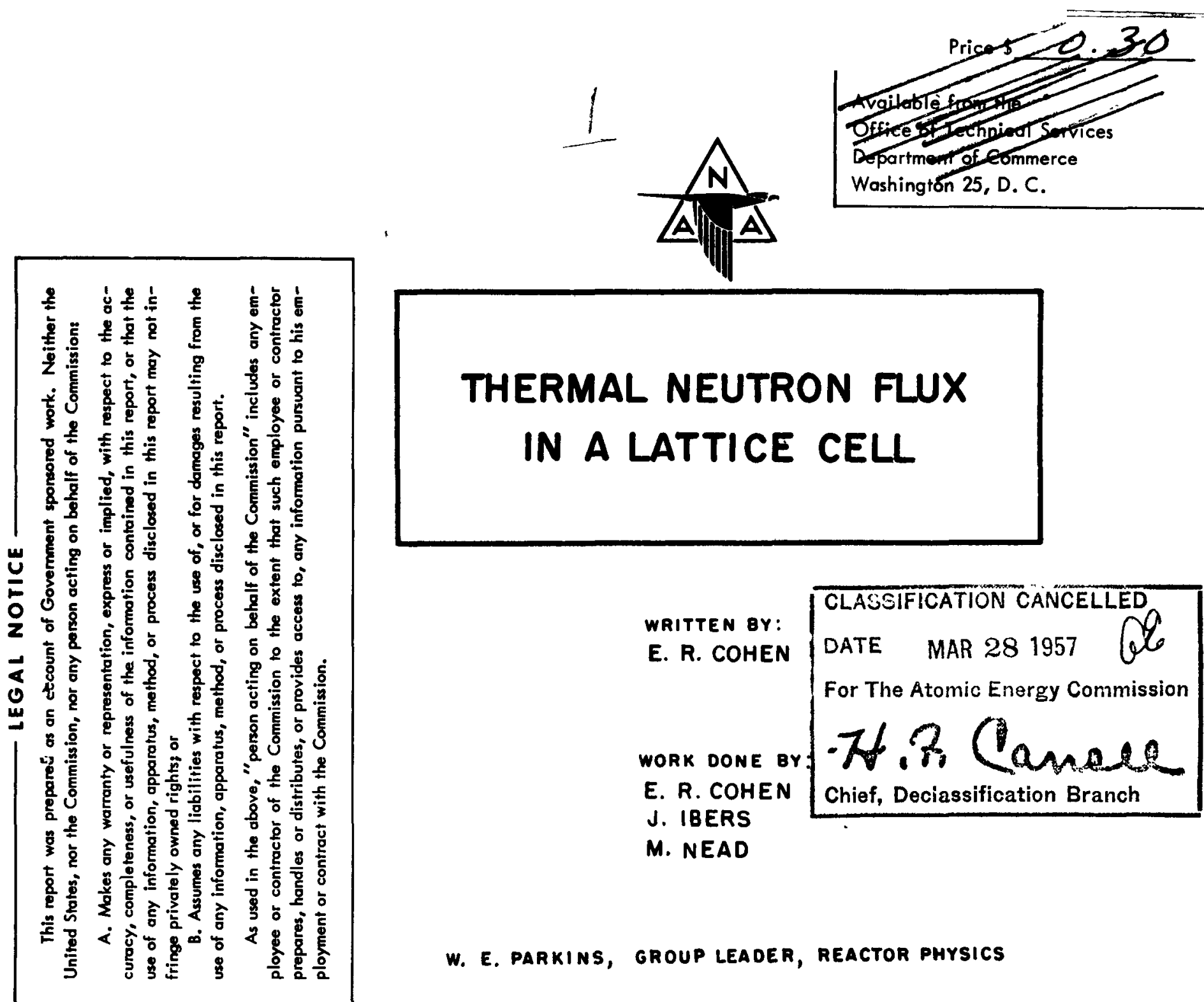

\section{THERMAL NEUTRON FLUX IN A LATTICE CELL}

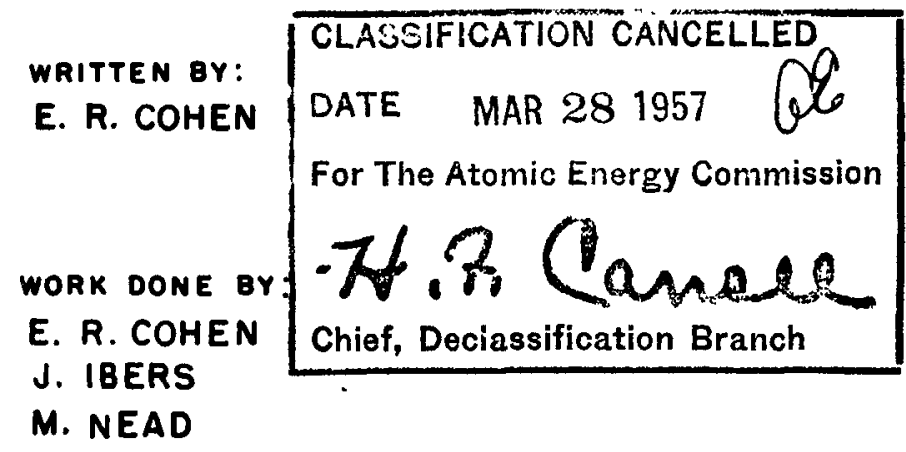

W. E. PARKINS, GROUP LEADER, REACTOR PHYSICS

S. SIEGEL-ASSOCIATE DIRECTOR

C. STARR - DIRECTOR

\section{ATOMIC ENERGY RESEARCH DEPARTMENT NORTHAMERICAN AVIATION, INC. P. O. BOX 309




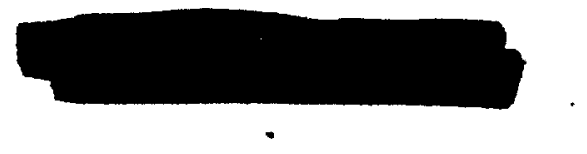

-

.

4

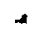

I

,
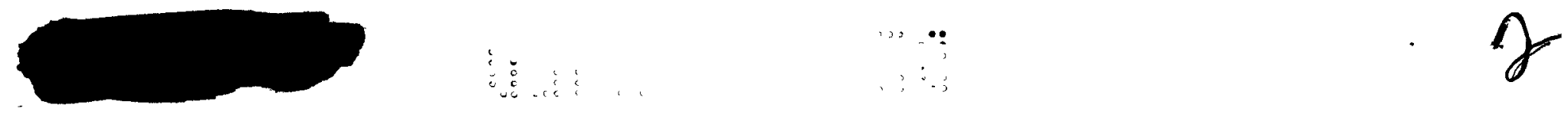


\section{DISCLAIMER}

This report was prepared as an account of work sponsored by an agency of the United States Government. Neither the United States Government nor any agency Thereof, nor any of their employees, makes any warranty, express or implied, or assumes any legal liability or responsibility for the accuracy, completeness, or usefulness of any information, apparatus, product, or process disclosed, or represents that its use would not infringe privately owned rights. Reference herein to any specific commercial product, process, or service by trade name, trademark, manufacturer, or otherwise does not necessarily constitute or imply its endorsement, recommendation, or favoring by the United States Government or any agency thereof. The views and opinions of authors expressed herein do not necessarily state or reflect those of the United States Government or any agency thereof. 


\section{DISCLAIMER}

Portions of this document may be illegible in electronic image products. Images are produced from the best available original document. 


\section{ABSTRACT}

The distribution of neutrons in the moderator of an infinite squarelattice array is found, assuming diffusion theory and a uniform production of neutrons throughout the moderator. The variation of neutron flux density along the sides of a lattice cell is shown to be significant. In spite of this, the thermal utilization of the lattice can be quite accurately calculated by the use of a "cylindricalized" cell. The absorption of neutrons by the moderator is shown to have an effect on the flux distribution which is negligible if the cell spacing is smaller than the thermal diffusion length of the moderator. (aullaj)

This report is based on studies conducted for the Atomic Energy Commission under Contract AT-11-1-GEN-8. 


\section{INTRODUCTION}

The usual calculation of thermal utilization ${ }^{l}$ in a heterogeneous reactor is based on a flux distribution in the moderator derived on the assumption of a "sphericized" or "cylindricalized" cell. The actual lattice cell, which may be (in the most common arrangements) square or hexagonal, is replaced by a cylinder with the same cross sectional area, and in this cylindrically symmetric situation the neutron diffusion current is made to vanish at the edge of the "cell" rather than the correct boundary condition which is that there is no diffusion current normal to the actual cell boundaries.

In this paper we shall obtain a solution for the neutron flux in a square lattice cell in which the correct boundary conditions are applied along the sides of the square. The approximation of one-velocity, elementary diffusion theory is maintained. The only incorrectly applied boundary condition is at the surface of the fuel rod but the inaccuracy vanishes as the radius of the rod becomes small and the inaccuracy is negligible even if the diameter of the rod is half the spacing between rods.

\section{SOLUTION OF THE DIFFUSION EQUATION}

We shall assume that the neutron flux, $\phi$, in the moderator satisfies the equation

$$
\nabla^{2} \phi-\frac{1}{L^{2}} \phi=-\frac{3}{\lambda} s_{0}
$$

where $L^{2}$ is the diffusion length in the moderator; $\lambda$, the transport mean free path in the moderator and $s_{0}$ the thermal source density, assumed to be constant over the moderator.

The geometry of the problem is indicated in Fig. 1. Uranium rods are spaced in a square array with lattice spacing, a. If the coordinate system is chosen so that the centers of the rods are at $x=K_{1} a, y=K_{2} a$ (where $K_{1}$ and $\mathrm{K}_{2}$ are arbitrary integers) and we consider the region near the rod at the origin, the symmetry of the problem imposes the conditions that the lines 
$x= \pm \frac{a}{2}$ and $y= \pm \frac{a}{2}$ are symmetry lines of the flux distribution.

We obtain a solution of Eq. (1) by separation of variables ${ }^{2}$ and write

$$
\begin{array}{rlrl}
\phi & =\sum_{n=0}^{\infty} A_{n} \cos \frac{2 n \pi x}{a} \cosh \varkappa_{n}\left(\frac{a}{2}-y\right)+\frac{3 L^{2}}{\lambda} s_{0} & 0<y<a \\
& =\sum_{n=0}^{\infty} A_{n} \cos \frac{2 n \pi x}{a} \cosh x_{n}\left(\frac{a}{2}+y\right)+\frac{3 L^{2}}{\lambda} s_{0} & -a<y<0
\end{array}
$$

The form of this solution has been chosen so as to satisfy the symmetry and periodicity conditions. The values of the constants $\varkappa_{n}$ are determined by putting $E_{q}$. (2) back into the differential equation and we find

$$
\mathcal{H}_{\mathrm{n}}^{2}=\left(\frac{2 \mathrm{n} \pi}{\mathrm{a}}\right)^{2}+\frac{1}{\mathrm{~L}^{2}}
$$

There is still the boundary condition due to the absorption of the fuel rod. We assume that in the moderator the effect of the fuel rod can be represented by a line sink at the origin. It is well known in potential theory that in the equivalent electrostatic problem such an approximation is valid if the actual radius of the sink is small; the exact effect of the rod could be obtained by including higher order terms corresponding to dipole, quadrupole, etc. In the present problem the symmetry of the boundary conditions is such that the first nonzero correction term of this kind is the quadrupole distribution. We can therefore assume that the simple line sink will be a reasonably valid approximation. We shall carry out the solution on this basis and then look at it to see how well it might be expected to agree with reality and over what range of rod radius the solution is valid.

We shall determine the constants $A_{n}$ in the standard procedure for finding the Green's function. The flux density has been defined separately for $y<0$ and $y>0$. We must match these two solutions so that they represent a continuous function throughout the moderator. The constants in the two solutions have already been chosen so that the flux density itself is continuous across the boundary. The current must also be continuous everywhere except possibly at the singularity. The neutron current in the region $y>0$ is

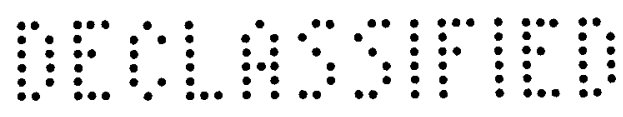




$$
j_{+}(y)=\frac{\lambda}{3} \sum_{n=0}^{\infty} A_{n} \varkappa_{n} \cos \frac{2 n \pi x}{a} \sinh \varkappa_{n}\left(\frac{a}{2}-y\right)
$$

and for $y<0$

$$
j_{-}(y)=\frac{-\lambda}{3} \sum_{n=0}^{\infty} A_{n} \mathscr{H}_{n} \cos \frac{2 n \pi x}{a} \sinh \mathscr{H}_{n}\left(\frac{a}{2}+y\right) .
$$

There is therefore a discontinuity in the current across the plane $y=0$ given by

$$
\left\{j_{+}(0)-j_{-}(0)\right\}=\frac{2 \lambda}{3} \sum_{n=0}^{\infty} A_{n} \varkappa_{n} \sinh \frac{\mathcal{H}_{n} a}{2} \cos \frac{2 n \pi x}{a}
$$

The number of neutrons absorbed per unit length of the line $y=0$ (and therefore also the discontinuity in the current) is zero except at the point $x=0$ where the line singularity absorbs, say, $Q$ neutrons per second; therefore

$$
\left\{j_{+}(0)-j_{-}(0)\right\}=-Q \delta(x)
$$

where $\delta(x)$ is the Dirac delta function. We therefore evaluate $A_{n}$ by the usual Fourier expansion

$$
\begin{gathered}
-Q \int_{-\mathrm{a} / 2}^{\mathrm{a} / 2} \delta(\mathrm{x}) \cos \frac{2 \mathrm{~m} \pi \mathrm{x}}{\mathrm{a}} \mathrm{dx}=\frac{2 \lambda}{3} \sum_{\mathrm{n}} \mathrm{A}_{\mathrm{n}} \varkappa_{\mathrm{n}} \sinh \frac{\mathcal{H}_{\mathrm{n}} \mathrm{a}}{2} \int_{-\mathrm{a} / 2}^{\mathrm{a} / 2} \cos \frac{2 \mathrm{n} \pi \mathrm{x}}{\mathrm{a}} \cos \frac{2 \mathrm{~m} \pi \mathrm{x}}{\mathrm{a}} \mathrm{dx} \\
-\mathrm{Q}=\frac{2 \lambda}{3} \mathrm{~A}_{\mathrm{m}} \mu_{\mathrm{m}} \sinh \frac{\mathcal{L}_{\mathrm{m}} \mathrm{a}}{2} \cdot \frac{1+\delta_{\mathrm{mo}}}{2} \cdot \mathrm{a}
\end{gathered}
$$

or

$$
A_{m}=-\frac{3 Q}{2 \lambda\left(1+\delta_{m o}\right)} \cdot \frac{1}{\frac{\mu_{m}^{a}}{2} \sinh \frac{\mu_{m}^{a}}{2}}
$$

where $\delta_{\text {mo }}$ is the Kronecker delta symbol

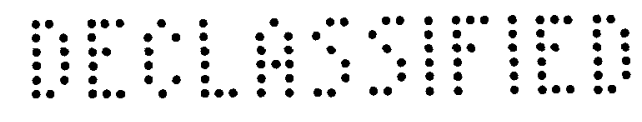




$$
\delta_{\text {mo }}=\begin{array}{ll}
1 & m=0 \\
0 & m \neq 0
\end{array}
$$

We have thus evaluated all the constants in the solution and inserting these into Eq. (2) gives the flux distribution in a square lattice cell. The flux is therefore:

$$
\phi=\frac{3 L^{2} s_{0}}{\lambda}-\frac{3 Q L}{2 \lambda a} \frac{\cosh \frac{a}{2 L}\left(1-\frac{2 y}{a}\right)}{\sinh \frac{a}{2 L}}-\frac{3 Q}{2 \lambda} \sum_{n=1} \frac{\cos \frac{2 n \pi x}{a} \cosh \varkappa_{n}\left(\frac{a}{2}-|y|\right)}{\frac{\mu_{n} a}{2} \sinh \frac{\mu_{n}{ }^{a}}{2}}
$$

\section{FLUX IN A NON-ABSORBING MODERATOR}

If we consider a non-absorbing moderator, $L \rightarrow \infty$ and we have from Eq. (3), $x_{n} a=2 n \pi$. The first two terms in Eq. (10) however both become infinitely large and we must take some care in evaluating the limit. In fact since there is no absorption in the moderator, the absorption in the sink, $Q$, must be equal to the total production $s_{0} a^{2}$ so that the first two terms approach the indeterminant form.

$$
\frac{3 L^{2}}{\lambda}\left(s_{0}-\frac{Q}{a^{2}}\right)=\frac{s_{0} a^{2}-Q}{a^{2} \Sigma_{m}}=\frac{0}{0}
$$

$\left(\Sigma_{\mathrm{m}}=\right.$ macroscopic absorption cross section of moderator $)$. If we are more careful we expand the hyperbolic functions in power series, retaining the first two terms and obtain the expression:

$$
\phi=\mathrm{K}+\frac{3 \mathrm{Q}}{4 \pi \lambda} \Psi(\mathrm{x}, \mathrm{y})
$$

where $K$ is a constant still to be determined and

$$
\Psi(x, y)=\frac{\pi}{6}-\frac{\pi}{2}\left(1-\frac{2|y|}{a}\right)^{2}-2 \sum_{n=1} \frac{\cos \frac{2 n \pi x}{a} \cosh n \pi\left(1-\frac{2|y|}{a}\right)}{n \sinh n \pi}
$$


A contour plot of this function is given in Fig. 2. One striking feature is the extent to which the contour lines are circular even out to half the size of the cell. This makes it possible to fit boundary conditions to an actual rod quite accurately as long as the rod is not larger than a/2 in diameter. (Fitting a finite size rod into the solution for a line sink is similar to the analogous electrostatic problem with a dielectric cylinder).

We evaluate the constants $K$ and $Q$ by considering the boundary conditions at the surface of the fuel rod. The two conditions are that the net flow of neutrons into the fuel rod must be equal to the thermal neutron production in the moderator as well as being equal to the absorption in the fuel rod. To do this we must evaluate the flux and its radial derivative at the surface of the fuel rod. The form of Eq. (12) makes this difficult and it is easier, as well as instructive, to find an approximate form for $\phi$ which is valid near the rod. We can see from the contour plot of the function that near the origin $\Psi(x, y)$ is a function only of the radius $r$. We can therefore simplify our task by expanding $\Psi(\mathrm{x}, \mathrm{y})$ along any convenient radius vector instead of carrying out a general two dimensional expansion in $x$ and $y$. We choose to make this expansion along the $x$-axis $(y=0)$ in which case we find

$$
\Psi(x, 0)=-\frac{\pi}{3}-2 \sum_{n=1} \frac{\operatorname{coth} n \pi}{n} \cos \frac{2 n \pi x}{a}
$$

The summation is slowly convergent; we can improve the convergence by substracting from it a sum with the following properties:

(a) The series to be subtracted has a readily recognizable sum.

(b) The terms of the series approach the terms of the series of Eq. (13).

One series which satisfies these conditions is

$$
\sum_{n=1} \frac{\cos \frac{2 n \pi x}{a}}{n}=-\ln \left(2 \sin \frac{\pi x}{a}\right)
$$


Eq. (14) can be established by expanding $\ln (1+z)$ as a power series in $z$ and equating real and imaginary parts with $z=\exp \{2 \pi i x / a\}$. We can then write

$$
\Psi(x, 0)=-\frac{\pi}{3}+2 \ln \left(2 \sin \frac{\pi x}{a}\right)-2 \sum \frac{\operatorname{coth} n \pi-1}{n} \cos \frac{2 n \pi x}{a}
$$

and expanding this for $x / a \ll 1$ we find

$$
\begin{aligned}
\Psi(x, 0)= & -\frac{\pi}{3}+2 \ln \frac{2 \pi x}{a}+2 \ln \left(1-\frac{\pi^{2} x^{2}}{6 a^{2}}+\ldots\right) \\
& -2 \sum_{n=1} \frac{\operatorname{coth} n \pi-1}{n}+\frac{4 \pi^{2} x^{2}}{a^{2}} \sum_{n=1} n(\operatorname{coth} n \pi-1)+\ldots \\
\phi(x, 0)= & K-\frac{3 Q}{4 \pi \lambda}\left\{\frac{\pi}{3}+2 \sum_{n=1} \frac{\operatorname{coth} n \pi-1}{n}\right\}+\frac{3 Q}{2 \pi \lambda} \ln \frac{2 \pi x}{a} \\
& -\frac{3 Q}{4 \pi \lambda} \cdot \frac{x^{2}}{a^{2}}\left\{\frac{\pi^{2}}{3}-4 \pi^{2} \sum_{n=1} n(\operatorname{cothn} n-1)\right\}+\ldots
\end{aligned}
$$

On the basis of the symmetry exhibited in Fig. 2 we can replace $x$ by $r$ and write

$$
\phi(x, 0)=\phi(r)
$$

The net flow of neutrons into the fuel rod of radius $r_{0}$ is

$$
\left.2 \pi \mathrm{r} \frac{\lambda}{3} \frac{\mathrm{d} \phi}{\mathrm{dr}}\right|_{\mathrm{r}_{\mathrm{o}}}=\mathrm{Q}\left[1-\frac{\pi \mathrm{r}_{\mathrm{o}}}{\mathrm{a}^{2}}\left\{\frac{\pi}{3}-4 \pi \sum \mathrm{n}(\operatorname{coth} \mathrm{n} \pi-1)\right\}\right]
$$

The production of thermal neutrons in the moderator is equal to the production per unit area times the area of the moderator or 


$$
\text { Production }=s_{0}\left(a^{2}-\pi r_{0}^{2}\right)=Q\left(1-\frac{\pi r_{0}^{2}}{a^{2}}\right)
$$

Equating (17) and (18) gives us the sum of the infinite series

$$
\sum_{n=1} n(\operatorname{coth} n \pi-1)=\frac{\pi-3}{12 \pi}
$$

(So far no more direct method for the evaluation of this series has been obtained. Its numerical value is 0.003755860 ...). Eq. (19) is not an evaluation of a constant but an indication of the internal consistency of the theory. That the production in the moderator is equal to the net flow of neutrons out of the moderator can of course be deduced directly from the differential equation.

The absorption in the fuel rod must also be related to the thermal flux in the fuel rod and its absorption cross section. If we consider the flux to be normalized to unity at the surface of the rod the absorption can be written as $\Sigma_{\mathrm{o}} \mathrm{V}_{\mathrm{o}} / \mathrm{F}$, where $\Sigma_{\mathrm{o}}$ is the fuel absorption cross section, $V_{0}$ the fuel volume per unit length and $F$ is the rod disadvantage factor: the ratio of the flux at the surface to the average flux throughout the rod.* We can define $F$ in this way without any need to specify further the form of $F$ or any assumption as to the applicability of diffusion theory inside the rod. Equating the absorption to the net flow of neutrons into the rod

$$
\Sigma_{0} V_{0} / F=Q\left(1-\frac{\pi r_{0}^{2}}{a^{2}}\right), \quad Q=\frac{\Sigma_{0} V_{0}}{F} \cdot \frac{a^{2}}{a^{2}-\pi r_{0}^{2}}
$$

* If the fuel rod is clad with a sheath whose macroscopic absorption cross section is $\Sigma_{1}$ and whose volume is $V_{1}$, the absorption term is replaced by $\frac{\sum_{\mathrm{o}} \mathrm{V}_{\mathrm{o}}}{\mathrm{F}}+\sum_{\mathrm{l}} \mathrm{v}_{\mathrm{l}}$. 
and evaluating $\mathrm{K}$ such that $\phi\left(\mathrm{r}_{\mathrm{o}}\right)=1$ gives us

$$
\mathrm{K}=1+\frac{3 \mathrm{Q}}{4 \pi \lambda}\left\{\frac{\pi}{3}+2 \sum \frac{\operatorname{coth} \mathrm{n} \pi-1}{\mathrm{n}}-\ln \frac{\pi \mathrm{r}_{\mathrm{o}}^{2}}{\mathrm{a}^{2}}-\ln 4 \pi+\frac{\pi \mathrm{r}_{\mathrm{o}}^{2}}{\mathrm{a}^{2}}\right\}
$$

and putting these values into Equation (12) we have

$$
\begin{aligned}
& \phi(x, y)=1+\frac{3 \sum_{0} v_{0}}{4 \pi \lambda F} \cdot \frac{a^{2}}{a^{2}-\pi r_{o}^{2}}\left\{\ln \frac{a^{2}}{\pi r_{o}^{2}}+\frac{\pi r_{o}^{2}}{a^{2}}-1.47634+\Psi(x, y)\right\} \\
& \phi(x, y) \approx 1+\frac{3 \sum_{o} v_{0}}{4 \pi \lambda F} \cdot \frac{a^{2}}{a^{2}-\pi r_{o}^{2}}\left\{2 \ln \frac{r}{r_{0}}-\frac{\pi\left(r^{2}-r_{o}^{2}\right)}{a^{2}}\right\} \quad r<a / 4 \\
& \left(r^{2}=x^{2}+y^{2}\right)
\end{aligned}
$$

\section{COMPARISON OF S QUARE CELL AND 'CYLINDR ICALIZED' CELL}

We shall now indicate the extent to which the square cell solution deviates from the solution using "cylindricalized" cells. In this approximation we replace the square cell boundary by a cylindrical boundary centered on the fuel rod and containing the same area as the square cell. The fact that such a cell is not "space-filling' is neglected and the assumption is made that the boundary condition of zero current can be applied acrass the circular boundary. This actually imposes this condition at points which are too close to the fuel rod near the cell corners and too far away from the fuel rod near the centers of the cell sides (Fig. 1). 
The differential equation, for $L^{2} \rightarrow \infty$, is

$$
\nabla^{2} \phi=-\frac{3}{\lambda} s_{0}
$$

and if we consider cylindrical symmetry

$$
\begin{aligned}
\frac{d}{d r}\left(r \frac{d \phi}{d r}\right) & =-\frac{3 s_{o} r}{\lambda} \\
r \frac{d \phi}{d r} & =\frac{3 s_{0}}{2 \lambda}\left(R^{2}-r^{2}\right)
\end{aligned}
$$

where the constant of integration has been chosen so as to satisfy the condition that the flux shall have a zero gradient at the cell boundary $(r=R)$. Integrating once more and chosing the flux to be unity at the surface of the rod $\left(r=r_{0}\right)$,

$$
\phi=1+\frac{3 s_{0}}{2 \lambda}\left[R^{2} \ln \frac{r}{r_{o}}-\frac{r^{2}-r_{o}^{2}}{2}\right]
$$

and expressing the source strength in terms of the absorption in the rod as in Eqs. (18) and (20)

$$
\phi=1+\frac{3 \sum_{0} V_{0}}{4 \pi \lambda F} \frac{R^{2}}{R^{2}-r_{o}^{2}}\left[2 \ln \frac{r}{r_{0}}-\frac{r^{2}-r_{o}^{2}}{R^{2}}\right]
$$

This expression is exactly the same as the expression for the square lattice cell geometry near the fuel rod given in Eq. (23) if we make the identification $\pi \mathrm{R}^{2}=\mathrm{a}^{2}$, which is just the condition that the "cylindricalized" cell has the same area as the square cell. Fig. 3 indicates just how the equivalent cell distribution solution compares with the square cell solution. $\Psi_{1}$ is the value of $\Psi(x, y)$ along a line through the origin perpendicular to the side of the cell; $\Psi_{2}$ is the value of $\Psi(\mathrm{x}, \mathrm{y})$ along a diagonal of the cell. $\Psi_{0}$ is the equivalent function associated with the flux distribution of $\mathrm{Eq} .(26)$. The curve $\Psi_{\mathrm{S}}$ is the value of $\Psi$ along the side of a lattice cell. This is approximately a cosine distribution as indicated by the dotted curve which is a simple cosine curve joining points $A$ and $B$. 
The neutron flux density at the edge of the equivalent cylindrical cell is

$$
\phi=1+\frac{3 \sum_{\mathrm{O}} \mathrm{V}_{\mathrm{O}}}{4 \pi \lambda \mathrm{F}}: \frac{\mathrm{R}^{2}}{\mathrm{R}^{2}-\mathrm{r}_{\mathrm{o}}^{2}}\left[\ln \frac{\mathrm{R}^{2}}{\mathrm{r}_{\mathrm{o}}^{2}}+\frac{\mathrm{r}_{\mathrm{o}}^{2}}{\mathrm{R}^{2}}-1\right]
$$

and comparing this with the flux at points A and B (see Fig. 3 and Eq. (22))

at $\mathrm{A}: \quad \phi=1+\frac{3 \sum_{\mathrm{o}} \mathrm{V}_{\mathrm{o}}}{4 \pi \lambda \mathrm{F}} \cdot \frac{\mathrm{a}^{2}}{\mathrm{a}^{2}-\pi \mathrm{r}_{\mathrm{o}}^{2}}\left[\ln \frac{\mathrm{a}^{2}}{\pi \mathrm{r}_{\mathrm{o}}^{2}}+\frac{\pi \mathrm{r}_{\mathrm{o}}^{2}}{\mathrm{a}^{2}}-0.78310\right]$

at $B: \quad \phi=1+\frac{3 \sum_{0} v_{0}}{4 \pi \lambda F} \cdot \frac{a^{2}}{a^{2}-\pi r_{o}^{2}}\left[\ln \frac{a^{2}}{\pi r_{o}^{2}}+\frac{\pi r_{o}^{2}}{a^{2}}-1.12977\right]$

\section{EFFECT OF ABSORPTION IN MODERATOR ON FLUX DISTRIBUTION}

In the previous sections the flux distribution was investigated for the case of zero absorption in the moderator. We shall now evaluate the order of magnitude of the correction to the flux distribution produced by a non-vanishing absorption cross section. We shall consider $\mathrm{a}^{2} / \mathrm{L}^{2}$ to be a small quantity in Eq. (10) and evaluate the first order terms. It will be shown that for any reasonable lattice configuration the absorption effect distorts the difference between the flux at the rod surface and the flux at the maximum (at the cell corner) by less than 1 per cent.

$a^{2} / L^{2}$ we find

If we return to Eq. (10), set $y=0$ and expand the flux as a series in

$$
\phi(x, 0)=\frac{3 L^{2}}{\lambda a^{2}}\left(s_{o} a^{2}-Q\right)+\frac{3 Q}{4 \pi \lambda}\left[\Psi(x, 0)+\frac{\pi a^{2}}{180 L^{2}}\right.
$$

$$
\left.+\frac{a^{2}}{4 L^{2}} \sum\left\{\frac{\operatorname{coth} n \pi}{n^{3} \pi^{3}}+\frac{1}{(n \pi \sinh n \pi)^{2}}\right\} \cos \frac{2 n \pi x}{a} . .\right]
$$


The last term in the brackets of Eq. (27) is certainly bounded in absolute value by its value at $\mathbf{x}=0$, hence it can be written as

$$
\begin{aligned}
& \frac{a^{2}}{4 L^{2}} g(x) \sum_{n=1}\left\{\frac{\operatorname{coth} n}{n^{3} \pi^{3}}+\frac{1}{(n \pi \sinh n \pi)^{2}}\right\} \\
& =\frac{0.00990 a^{2}}{L^{2}} g(x) \quad-1<g(x) \leqslant g(0)=1
\end{aligned}
$$

If we calculate the net neutron current into the rod of radius $r_{0}$ we obtain

$$
\begin{gathered}
\left.2 \pi r_{0} \frac{\lambda}{3} \frac{d \phi}{d r}\right|_{r_{0}}=\frac{Q}{2}\left\{\left.r_{0} \frac{d \Psi}{d r}\right|_{r_{0}}+\frac{0.00990 a^{2}}{L^{2}} r_{0} g^{\prime}\left(r_{0}\right)\right\} \\
r_{0} \frac{d \Psi}{d r_{0}} \approx 2 \frac{a^{2}-\pi r_{0}^{2}}{a^{2}}
\end{gathered}
$$

and since this must be equal to the absorption in the fuel rod

$$
\frac{\sum_{0} V_{0}}{F}=Q\left[1-\frac{\pi r_{o}^{2}}{a^{2}}+\frac{0.00990 a^{2}}{L^{2}} \cdot \frac{r_{o} g^{\prime}\left(r_{o}\right)}{2}\right]
$$

where the flux at the surface of the rod is taken equal to unity and the average flux in the rod is $1 / F$. The flux is therefore 


$$
\begin{aligned}
& \phi(x, 0)=1+\frac{3 \sum_{0} v_{0}}{4 \pi \lambda F}\left[1-\frac{\pi r_{o}^{2}}{a^{2}}+\frac{0.00990 a^{2}}{L^{2}} \cdot \frac{r_{o} g^{\prime}\left(r_{o}\right)}{2}\right]^{-1} \\
& \cdot\left\{\Psi(x, 0)-\Psi\left(r_{o}\right)+\frac{0.00990 a^{2}}{L^{2}}\left(g(x)-g\left(r_{o}\right)\right)\right\}
\end{aligned}
$$

The functions $g(x)$ and $x^{\prime}(x)$ are plotted in Fig. 4. Now $\Psi(x, 0)-\Psi\left(r_{0}\right)$ is approximately equal to

$$
2 \ln \frac{x}{r_{o}}-\frac{\pi\left(x^{2}-r_{o}^{2}\right)}{a^{2}}
$$

which is the form obtained in the "cylindricalized" solution. The maximum value of the last term in the curly brackets is

$$
\frac{0.00990 \mathrm{a}^{2}}{\mathrm{~L}^{2}}(1.757)=\frac{0.01743 \mathrm{a}^{2}}{\mathrm{~L}^{2}}
$$

so that the relative error in the difference between maximum flux and flux at the surface of the rod due to a neglect of the absorption in the moderator is therefore approximately

$$
\delta=\frac{0.01743 \mathrm{a}^{2} / \mathrm{L}^{2}}{\ln \frac{\mathrm{a}^{2}}{\pi \mathrm{r}_{0}{ }^{2}}-1}
$$

For any reasonable graphite or heavy water moderated lattice, the 
denominator of the expression above lies between 1 and 5 , and $\delta$ will be of the order of 1 per cent even for the extreme case of $a=L$. Since a more realistic value is $a^{2} / L^{2} \approx 0.1$ we see that $\delta$ will in general be quite negligible.

\section{THERMAL UTILIZATION IN A SQUARE CELL}

The previous section has indicated that the distortion of the flux distribution due to absorption of neutrons in the moderator is small if $a<L$. The thermal utilization can therefore be calculated by using the flux distribution for a non-absorbing moderator and finding the absorption in the moderator by integrating the actual moderator absorption cross sections over this approximate flux distribution. This procedure will introduce a relative error of order of magnitude $\delta$ into the quantity $(1-f) / f$.

The thermal utilization is the ratio of absorption in the rod to the total absorption. The absorption in the fuel rod is $\sum_{0} v_{0} / F$ and the absorption in the moderator is

$$
\Sigma_{\mathrm{m}} \int_{(\mathrm{mod})} \phi(\mathrm{x}, \mathrm{y}) \mathrm{d} \mathbf{x} \mathrm{dy}
$$

where $\sum_{m}$ is the macroscopic absorption cross section of the moderator and the integration extends over the moderator only. One can then write

$$
\frac{1-f}{f}=\frac{\sum_{m} F}{\sum_{0} V_{0}} \iint_{(\bmod )} \phi(x, y) d x d y
$$

the calculation of $f$ is then reduced to an evaluation of the integral. The most convenient method of evaluation is to break the integral into two parts 
Actually, of course, the flux has not been found inside the rod; $\phi(x, y)$ refers however here to the forms given in Eq. (12) and $\mathrm{Eq}$. (23) which are defined analytically throughout the cell. The integral over the entire cell includes too much of the function $\phi(x, y)$ in the integration; the second integral then subtracts off that part which is not wanted.

$$
\begin{gathered}
\iint_{(\text {cell })} \phi(x, y) d x d y=\int_{-\frac{a}{2}}^{\frac{a}{2}} \int_{-\frac{a}{2}}^{\frac{a}{2}}\left[K+\frac{3 Q}{4 \pi \lambda} \Psi(x, y)\right] d x d y \\
=K a^{2}
\end{gathered}
$$

For the integral over the rod use Eq. (23):

$$
\begin{aligned}
& \iint_{(\mathrm{rod})} \phi(x, y) d x d y=\int_{0}^{r_{0}} \phi(x, y) 2 \pi r d r \\
& =\pi r_{0}^{2}+\frac{3 \sum_{0} v_{0}}{4 \pi \lambda F} \cdot \frac{a^{2}}{a^{2}-\pi r_{o}^{2}} \int_{0}^{r_{0}}\left\{2 \ln \frac{r_{0}}{r_{0}}-\frac{\pi\left(r^{2}-r_{o}^{2}\right)}{a^{2}}\right\} 2 \pi r d r
\end{aligned}
$$

We now make the substitution $u=\pi r^{2}, v_{0}=\pi r_{0}^{2}$, and

$$
\begin{aligned}
& \iint_{(\operatorname{rod})} \phi(x, y) d x d y=v_{o}+\frac{3 \sum_{0} v_{o}}{4 \pi \lambda F} \cdot \frac{a^{2}}{a^{2}-v_{o}} \int_{0}^{v} \cdot\left\{\ln _{v_{o}}-\frac{u-v_{o}}{a^{2}}\right\} d u \\
& =v_{0}+\frac{3 \sum_{0} v_{o}}{4 \pi \lambda F} \cdot \frac{a^{2}}{a^{2}-v_{o}}\left\{-v_{o}+\frac{v_{o}^{2}}{2 a^{2}}\right\}
\end{aligned}
$$


therefore

$$
\iint_{(\bmod )} \phi(x, y) d x d y=K a^{2}-v_{0}+\frac{3 \sum_{0} v_{0}}{4 \pi \lambda F} \cdot \frac{a^{2}}{a^{2}-v_{o}}\left\{v_{0}-\frac{v_{0}^{2}}{2 a^{2}}\right\}
$$

and inserting the value of $\mathrm{K}$ from $\mathrm{Eq}$. (21)

$$
\begin{aligned}
\iint_{(\mathrm{mod})} \phi(x, y) d x d y= & a^{2}-v_{0}+\frac{3 \sum v_{0}}{4 \pi \lambda F} \cdot \frac{a^{2}}{a^{2}-v_{0}}\left\{a^{2} \ln \frac{a^{2}}{v_{0}}+2 v_{0}-\frac{v_{0}^{2}}{2 a^{2}}\right\} \\
& +a^{2}\left(\frac{\pi}{3}+2 \sum \frac{\operatorname{coth} n \pi-1}{n}-\ln 4 \pi\right)
\end{aligned}
$$

Then, putting this integral into Eq. (34) we obtain the formula

$$
\begin{aligned}
& \frac{1-\mathrm{f}}{\mathrm{f}}=\frac{\sum_{\mathrm{m}} \mathrm{V}_{\mathrm{m}}}{\sum_{\mathrm{o}} \mathrm{V}_{\mathrm{o}}}+\frac{\mathrm{a}^{2}}{4 \pi \mathrm{L}^{2}}\left\{\frac{\mathrm{a}^{2}}{\mathrm{~V}_{\mathrm{m}}} \ln \frac{\mathrm{a}^{2}}{\mathrm{~V}_{\mathrm{o}}}-\frac{\mathrm{v}_{\mathrm{o}}^{2}}{2 \mathrm{a}^{2} \mathrm{~V}_{\mathrm{m}}}-1.47634 \frac{\mathrm{a}^{2}}{\mathrm{v}_{\mathrm{m}}}+\frac{2 \mathrm{~V}_{\mathrm{o}}}{\mathrm{V}_{\mathrm{m}}}\right\} \\
& =\frac{\sum_{m} v_{m}}{\sum_{0} V_{o}} F+\frac{a^{2}}{4 \pi L^{2}}\left\{\frac{a^{2}}{v_{m}} n \frac{a^{2}}{v_{o}}-1-\frac{v_{m}}{2 a^{2}}+0.02366 \frac{a^{2}}{V_{m}}\right\}
\end{aligned}
$$

where $V_{0}=$ volume per unit length of fuel rod.

$$
V_{m}=a^{2}-V_{0}=\text { volume per unit length of moderator. }
$$$$
a^{2}=\text { volume per unit length of lattice cell. }
$$

This expression for thermal utilization is almost the same as the one obtained in the "cylindricalized" approximation with an equivalent cell radius $R=a / \sqrt{\pi} ;$ the only difference is the term 
NAP - SR - 155

Page 19

$$
0.02366 \frac{\mathrm{a}^{2}}{\mathrm{~V}_{\mathrm{m}}}
$$

which does not appear in the "cylindricalized" approximation. This term however contributes in general less than 1 per cent of the excess absorption term and is probably less important than the errors introduced into the theory by the assumption of a uniform thermal neutron source term throughout the moderator (an assumption which is not realistic if the area of the lattice cell is comparable with or larger than the Fermi age).

VII. CONCLUSIONS

The flux distribution in a square-lattice cell has been calculated exactly on the basis of diffusion theory, assuming a uniform production of thermal neutrons throughout the moderator. The results (inasmuch as they influence the calculation of thermal utilization) indicate that the usual approximation of "cylindricalized" cells gives quite accurate values; the differences in the values of $f$ calculated exactly and approximately are probably smaller than the varactions introduced by uncertainties in the numerical values of the physical constarts involved, the fact that diffusion theory is a poor approximation near the fuel rod, and the actual non-uniformity of the thermal sources in the moderator.

$\frac{7}{\frac{2}{\infty}}$

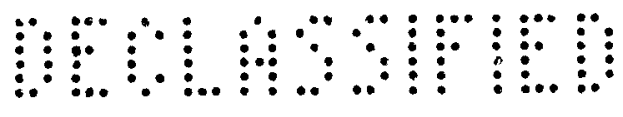




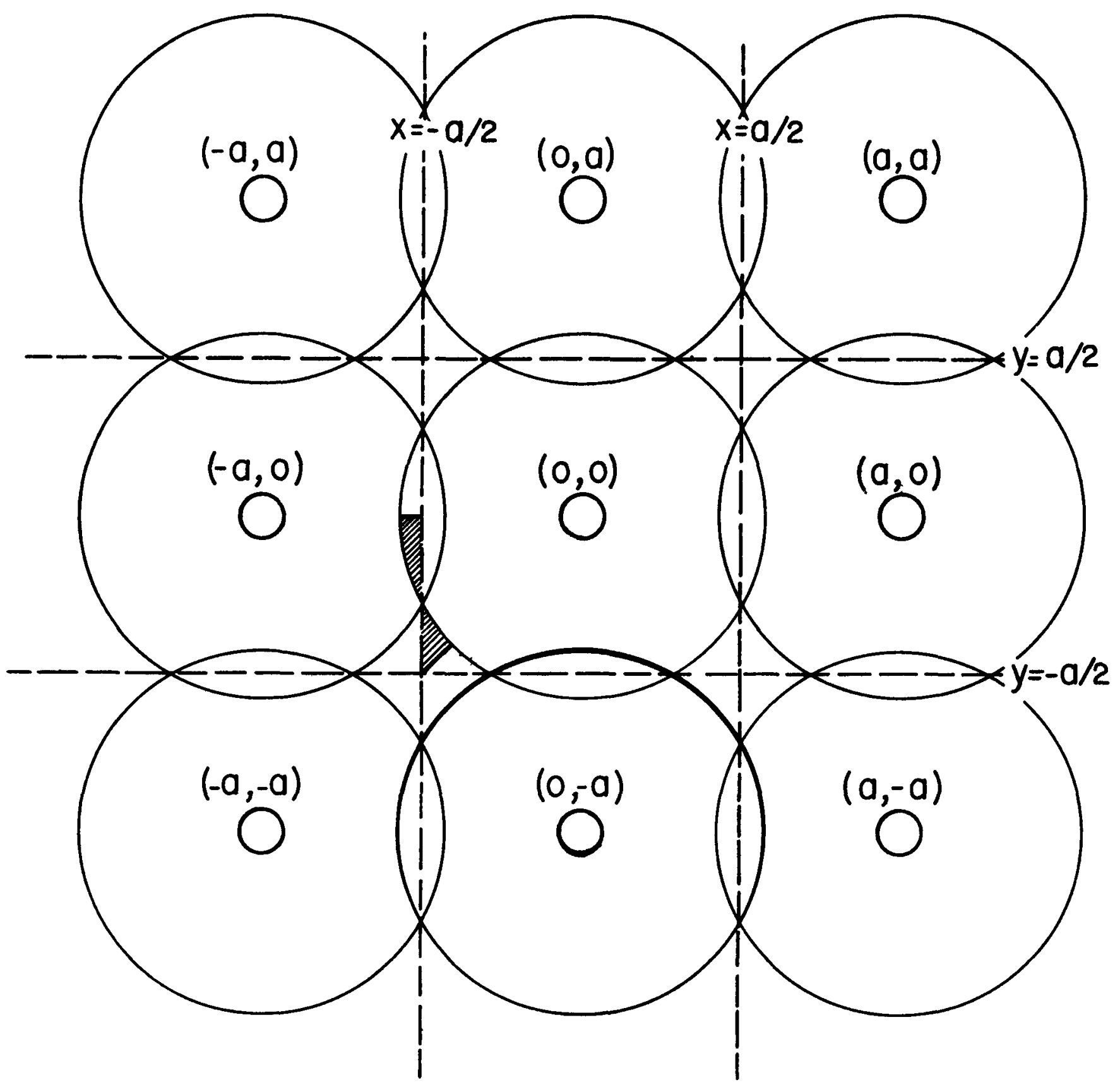

Figure 1. Lattice Showing Square Cells and Equivalent "Cylindricalized" Cells. The Two Shaded Areas Are Equal. 


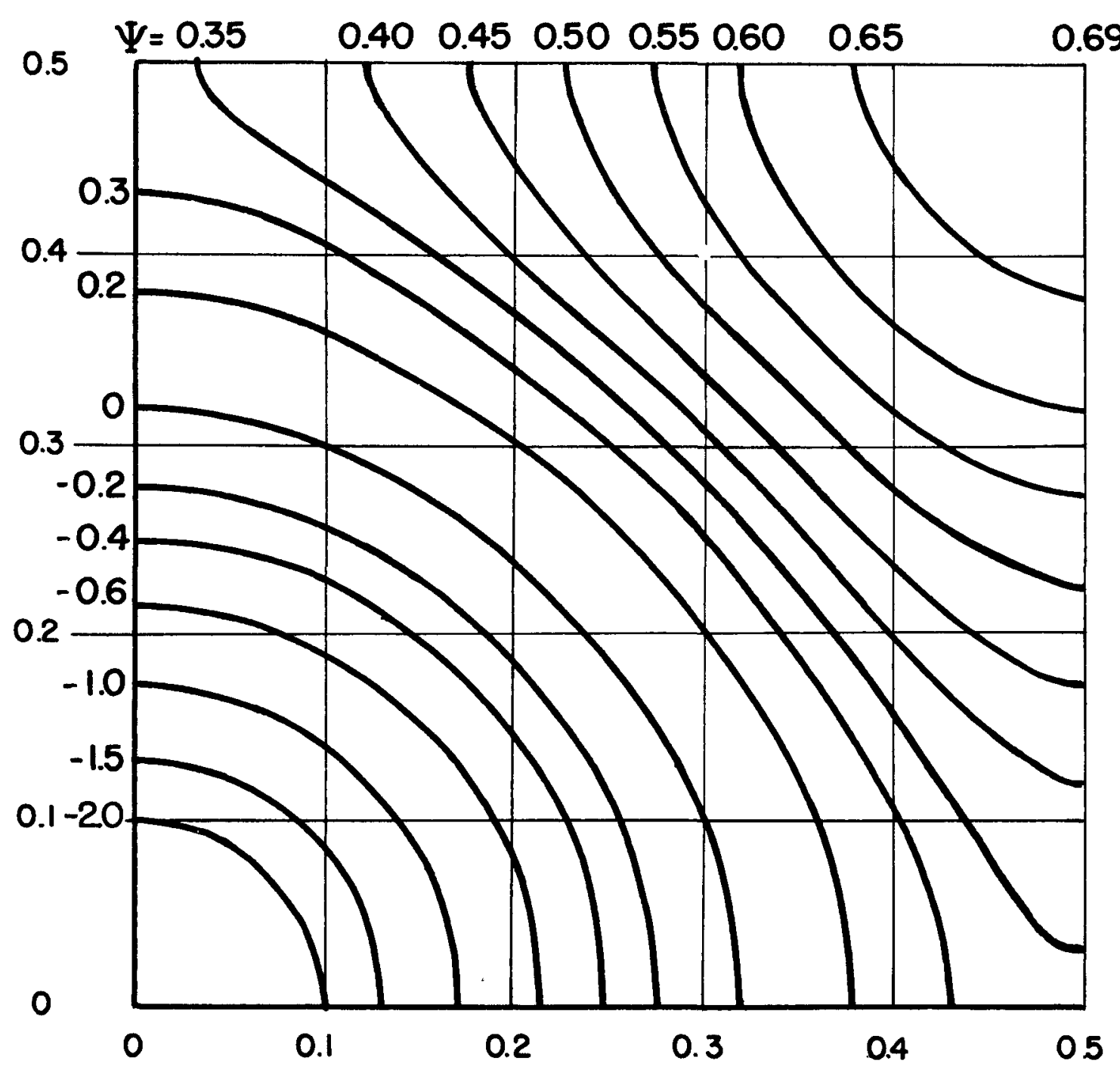

Figure 2. Contour Map of $\Psi(x, y)$ for One Quadrant of a Lattice Cell. The fuel rods are at the origin $(0,0)$ and at integral lattice points. 
23

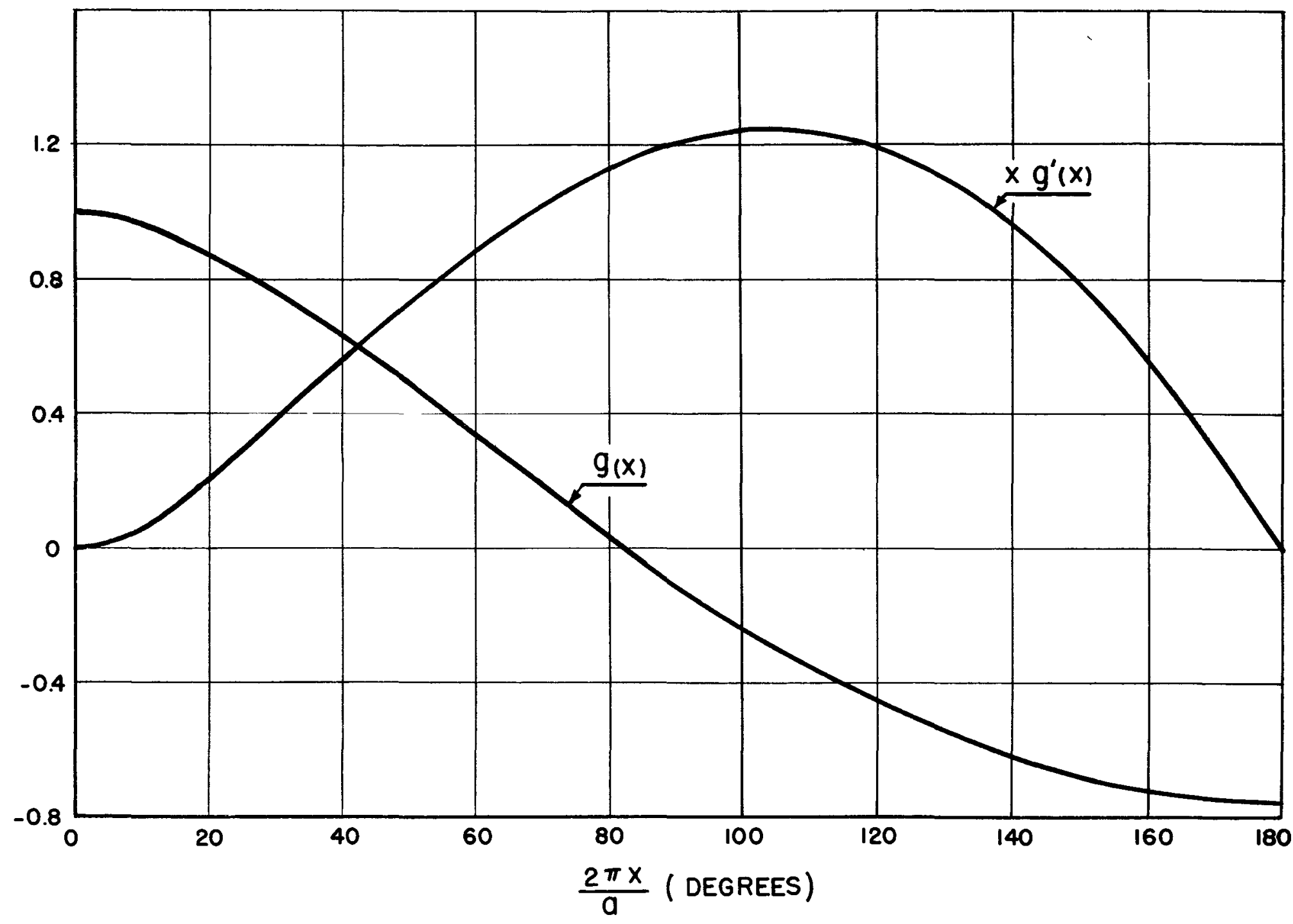

Figure 4. 


\section{REF ER ENCES}

1. Weinberg, A. M., Science and Engineering of Nuclear Power, Vol. II C. Goodman, editor; Aldison-Wesley, 1949. Chap. 6.

2. Jeffries, H. and B. S. Jeffries, Mathematical Physics, Cambridge, 1950. Chap. 18. 